\title{
Effects of anti-CD44 monoclonal antibody IM7 carried with chitosan polylactic acid-coated nano-particles on the treatment of ovarian cancer
}

\author{
YIZHUO YANG $^{1 *}$, XINGHUI ZHAO ${ }^{2 *}$, XIULI LI $^{1}$, ZHIFENG YAN $^{1}$, ZHONGYU LIU $^{1}$ and YALI LI ${ }^{1}$ \\ ${ }^{1}$ Department of Obstetrics and Gynecology, General Hospital of PLA; ${ }^{2}$ State Key Laboratory of Pathogens and Biosecurity, \\ Laboratory of Applied Molecular Biology, Beijing Institute of Microbiology and Epidemiology, Beijing 100853, P.R. China
}

Received June 2, 2015; Accepted October 11, 2016

DOI: $10.3892 / \mathrm{ol} .2016 .5413$

\begin{abstract}
Failure in early diagnosis and ineffective treatment are the major causes of ovarian cancer mortality. Hyaluronan and its receptor, cluster of differentiation (CD)44, have been considered to be valid targets for treating cancer. The anti-CD44 monoclonal antibody IM7 is effective in treating ovarian cancer; however, its toxicity should not be ignored. The present study has developed a new drug carrier system composed of chitosan nano-particles coated with polylactic acid (PLA) to improve the treatment efficacy and reduce toxicity. An ionic crosslinking method and 1-ethyl-3-(3-dimethylaminopropyl) carbodiimide hydrochloride/N-hydroxysuccinimide were used to prepare the IM7 antibody, which was loaded with chitosan nano-particles. The surfaces of the nano-particles were coated with PLA to generate PLA-chitosan-IM7. Subsequently, transmission electron microscopy (TEM) was used to observe the size and zeta potential of the nano-particles. In addition, a spectrophotometer was used to calculate the loading rate and release rate of the nano-particles in acidic and neutral environments. MTT assay was used to evaluate the anti-proliferative effect of PLA-chitosan-IM7 on the human ovarian cancer cell line HO-8910PM. In addition, an in vivo imaging system was used to further investigate the effect of PLA-chitosan-IM7 on the treatment of mice with ovarian cancer. A total of 35 days subsequent to PLA-chitosan-IM7 treatment, all animals were sacrificed by $\mathrm{CO}_{2}$, and the tumors were removed and weighted. The PLA-chitosan-IM7 nano-particles were successfully prepared, since TEM revealed that their size was $300-400 \mathrm{~nm}$ and their zeta potential was $+25 \mathrm{mV}$. According
\end{abstract}

Correspondence to: Dr Yali Li, Department of Obstetrics and Gynecology, General Hospital of PLA, 28 Fuxing Road, Beijing 100853, P.R. China

E-mail: yali_li88@126.com

*Contributed equally

Key words: ovarian cancer, anti-CD44 monoclonal antibody, IM7 antibody, chitosan nano-particles, polylactic acid to the spectrophotometry results, the loading rate was $52 \%$, and PLA-chitosan-IM7 exhibited good resistance to acids. MTT assay demonstrated that PLA-chitosan-IM7 could suppress the proliferation of HO-8910PM cells in vitro. The in vivo imaging system revealed that PLA-chitosan-IM7 was effective in controlling the development of human ovarian cancer cells and the tumor weight. These results suggest that PLA-chitosan-IM7 could be effective in treating cancers in vitro and in vivo, which may provide a novel approach to enhance the effectiveness of anti-CD44 treatment while reducing its toxicity.

\section{Introduction}

Ovarian cancer is the most lethal gynecological cancer, and the 5 -year survival rate of patients is only $30-50 \%$ (1). Failure in early diagnosis and ineffective treatment are the major causes of mortality. Therefore, recent research has been focused on the identification of new methods of early diagnosis and novel strategies for therapy (1).

Hyaluronan (HA) is a macromolecular glycosaminoglycan, which is the major component of the extracellular matrix and intercellular substance. HA serves essential roles due to its viscoelasticity, hydrability and interaction with its receptors. The presence and absence of HA binding proteins and whether HA is connected with its cell surface receptors decide its function. Increased HA was able to strengthen the capability of invasion, metastasis and proliferation in ovarian tumor models $(2,3)$. Cluster of differentiation (CD)44 is a cell surface transmembrane glycoprotein, which exhibits two forms: i) Standard CD44 (CD44S, also named CD44H), which is mainly expressed on the surface of stromal and hematopoietic cells; and ii) variant CD44 (CD44V), which is mainly expressed on the surface of epithelial cells and tumors (4). Ovarian cancers express both CD44S and CD44V. CD44 is expressed in a large number of cell types, including adhesion of cells and stromal components, lymphocyte homing, activation of $\mathrm{T}$ lymphocyte cells and signal transduction. Both CD44V and CD44S have been reported to be important in the development and metastasis of ovarian cancer and other gynecological tumors (4). The binding of HA to CD44 can initiate several different signaling pathways, including 
human epidermal growth factor receptor 2, c-Src kinase and extracellular signal-regulated kinase (5-12). The activation of these pathways may lead to increased motility, adhesion and invasion as well as tumor growth in ovarian cancer $(3,11,12)$. In summary, HA and its receptor CD44 are considered to be valid targets for the treatment of various types of cancer.

Several studies on the treatment efficacy and cytotoxic effect of chemotherapeutic drugs have been conducted, including paclitaxel, cisplatin and polyethylenimine (PEI) DNA particles, which suggested that HA-conjugation could be utilized as tumor-targeted therapy (13-15). Besides chemotherapy, numerous scholars have paid attention to biotherapy, including hyaluronidase enzymes, $\mathrm{PH} 20$ and pegylated recombinant human hyaluronidase PH20 $(16,17)$. Methods used to block the action of CD44 include neutralizing antibodies, small interfering RNA, antisense RNA, complementary DNA vaccination as well as the CD44 inhibitor silibinin. These methods have shown therapeutic effect, although their toxicity could not be ignored (17). Non-toxic alternative therapies remain to be further investigated.

Nano-particles are solid colloidal particles with a diameter of 10-500 nm, which is much smaller than that of a cell $(10-1,000 \mathrm{~nm})$. Due to this great advantage, these particles are easily absorbed by tissues and cells. As a new form of drug delivery and release, nano-particles appear to be promising, and have been widely studied in recent years (18). Chitosan, as a new type of nano-particle, has been reported to be relatively good in bioadhesivity and promotion of absorption as well as inhibition of enzymes (18). The IM7 antibody is one of the most important anti-CD44 monoclonal antibodies in mediating cell-cell and cell-matrix interactions through its affinity for HA. IM7 has been used to treat cancers; however, its major disadvantages are its toxicity and unsustainable stimulating effect (4).

In the present study, a new carrier system composed of chitosan nano-particles coated with polylactic acid (PLA) (termed PLA-chitosan-IM7) has been developed to carry IM7. The advantages and disadvantages of treating CD44-positive cancers in vitro and in vivo with PLA-chitosan-IM7 were evaluated, and the results obtained may provide a new approach for cancer treatment.

\section{Materials and methods}

Preparation of IM7 loaded with chitosan nano-particles. An ionic crosslinking method was used to prepare nano-particles according the method of Bodmeier (16). First, $200 \mu 1$ IM7 (Cat\#ab171211; Abcam, Cambridge, UK) were added to $4 \mathrm{ml}$ thiamine pyrophosphate (TPP) solution (Cat\#C8754; Sigma-Aldrich; Merck Millipore, Darmstadt, Germany) at $1 \mathrm{mg} / \mathrm{ml}$ (pH 7-9), and then the mixture was added to $10 \mathrm{ml}$ chitosan solution (Cat\#740,500; Sigma-Aldrich; Merck Millipore) ( $\mathrm{pH} 4-6)$ at a constant rotating speed, and incubated for $10 \mathrm{~min}$ at $57^{\circ} \mathrm{C}$. Due to molecular linkage between TPP and chitosan, the nano-particles were prepared when the color of the solution became homogeneously light blue. The size and zeta potential of the nano-particles was determined with a transmission electron microscope (TEM).

Surface coverage of chitosan nano-particles with PLA. 1-Ethyl-3-(3-dimethylaminopropyl) carbodiimide hydrochloride (EDC)/N-hydroxysuccinimide (NHS) was used to coat the chitosan nano-particles with PLA (Sigma-Aldrich; Merck Millipore). First, $0.4 \mathrm{mg}$ EDC (final concentration, 2 mM) (Cat\#22,890; Thermo Fisher Scientific, Inc., Waltham, MA, USA) and $0.6 \mathrm{mg}$ NHS (final concentration, $5 \mathrm{mM}$ ) (Cat\#24,500; Thermo Fisher Scientific, Inc.) were added to $1 \mathrm{ml}$ PLA, and then the mixture was added to the nano-particles solution. The reaction components were mixed thoroughly and allowed to react for $15 \mathrm{~min}$ at room temperature.

Investigation of drug loading rate and stability. An spectrophotometer was used to detect the optical density (OD) of IM7 prior and subsequent to being loaded with nano-particles, and the loading rate was calculated as follows: Drug loading rate = amount of doxorubicin (Dox; Sigma-Aldrich; Merck Millipore) encapsulated / total weight of nano-particles. In addition, the release rate of PLA-chitosan-IM7 was observed for $0,1,2,5,6$ and 7 days at neutral ( $\mathrm{pH} 7.4)$ and acidic (pH 5.0) environments, and was calculated as follows: Release rate $=$ amount of Dox encapsulated $/$ Total Dox added .

Anti-proliferative effect of PLA-chitosan-IM7 on an ovarian cancer line. The human ovarian cancer cell line HO-8910PM was purchased from Peking Union Medical College (Beijing, China) and cultured with RPMI 1640 medium with $10 \%$ fetal bovine serum (Thermo Fisher Scientific, Inc.) and 1\% penicillin/streptomycin. Then, MTT assay was used to observe the suppressing effect of PLA-chitosan-IM7 on HO-8910M cells. Briefly, HO-8910PM cells were cultured for $24 \mathrm{~h}$ and then divided into three groups: i) Control group without any treatment; ii) IM7 group treated with IM7 (final concentration, $20 \mathrm{ng} / \mathrm{ml}$ ); and iii) PLA group treated with PLA-chitosan-IM7 (final concentration of IM7, $20 \mathrm{ng} / \mathrm{ml}$ ). The stimulation time was 0, 12, 24, 36, 48 and 72 h. Lastly, MTT assay (Cat\#30-1010K; American Type Culture Collection, Manassas, VA, USA) was employed to observe the viability and proliferation of HO-8910PM as follows: Each group was adjusted to a density of $2 \times 10^{5}$ cells $/ \mathrm{ml}$ and plated into 96 -well culture plates. The plates were incubated for $6 \mathrm{~h}$, and $10 \mu \mathrm{l}$ MTT reagent was added until a purple precipitate was visible. Next, $10 \mu \mathrm{l}$ detergent reagent (dimethyl sulfoxide) was added and incubated at room temperature in the dark for $2 \mathrm{~h}$. The optical density was measured at $570 \mathrm{~nm}$ using a spectrophotometer.

Animal studies. In total, 15 female_BALB/c nude mice (6-8 weeks old; body weight, 20-22 g) were obtained from Vital River (Beijing, China) and housed under pathogen-free conditions with a $12 \mathrm{~h}$ light-dark cycle. Food and water were provided ad libitum throughout the study. The mice were subcutaneously injected with $2 \times 10^{6} \mathrm{HO}-8910 \mathrm{PM}$ cells for $72 \mathrm{~h}$ to successfully establish an ovarian mouse model. The 30 mice with ovarian cancer were divided into three groups: i) PLA group, which was treated with PLA-chitosan-IM7 at $100 \mathrm{ng} / \mathrm{g}$; ii) IM7 group, which was treated with IM7 antibody at $100 \mathrm{ng} / \mathrm{g}$; and iii) positive control group, which was treated with Dox at $50 \mathrm{ng} / \mathrm{g}$. Animal models of in vivo fluorescence were established according to the protocol of D-luciferin (Cat\#LUCNA; Gold BioTechnology, Inc., St. Louis, MO, USA). Each group received $15 \mathrm{mg} / \mathrm{ml} \mathrm{D}$-luciferin, and an in vivo imaging system (Xenogen IVIS spectrum; Caliper 
Life Sciences, Hopkinton, MA, USA) was used to evaluate the targeting specificity and treatment efficacy of IM7 loaded with PLA-chitosan nano-particles. In addition, when the mean volumes of tumors were between 150 and $200 \mathrm{~mm}^{3}$, mice were randomly divided in three groups (10 mice/group) prior to treatment. The tumor volume and body weight in each group were matched, at $180 \pm 10 \mathrm{~mm}^{3}$ and $20 \pm 2 \mathrm{~g}$, respectively.

A total of 35 days subsequent to PLA-chitosan-IM7 treatment, all mice were sacrificed by $\mathrm{CO}_{2}$, and the tumors were removed and weighted. All the animals experiments conducted in the present study were approved by the Animal Ethics Committee of General Hospital of PLA (Beijing, China).

Statistical analysis. All data were analyzed with SPSS 12.0 software (SPSS Inc., Chicago, IL, USA). Statistical analysis was performed using Student's $t$-test and $\mathrm{P}<0.05$ was considered to indicate a statistically significant difference.

\section{Results}

Preparation of PLA-chitosan-IM7 nano-particles. The preparation process is shown in Fig. 1. Upon preparation, TEM was used to observe the size and zeta potential of the nano-particles, and it was noticed t\$hat the diameter was 100-500 nm (mean value, $350 \mathrm{~nm}$ ) (Fig. 2A) and the zeta potential varied between -75 and $+45 \mathrm{mV}$ (Fig. 2B).

Determination of the loading rate and stability of PLA-chitosan-IM7. Spectrophotometry was used to determine the OD of IM7 prior and subsequent to being loaded, and the loading rate was calculated, which was $52 \%$. In addition, the stability of PLA-chitosan-IM7 was observed for 0, 1, 2, 5, 6 and 7 days at neutral ( $\mathrm{pH}$ 7.4) and acidic ( $\mathrm{pH}$ 5.0) environments. The results indicated that the release of PLA-chitosan-IM7 in acidic environments was slightly faster than that in neutral environments. PLA-chitosan-IM7 was stable at 3 days in any type of environment (Fig. 3).

Suppressing effect of PLA-chitosan-IM7 on an ovarian cancer cell line. MTT assay was used to analyze the influence of PLA-chitosan-IM7 nano-particles on the human ovarian cell line HO-8910PM. The results indicated that PLA-chitosan-IM7 and IM7 could suppress the proliferation of cancer cells ( $\mathrm{P}=0.0156$ vs. the control group). In addition, the survival time of the IM7 group was markedly lower than that of the other two groups, suggesting that application of IM7 alone had increased toxicity compared with PLA-chitosan-IM7 (Fig. 4).

Animal studies. An in vivo imaging system and a subcutaneous tumor model were employed to investigate the effect of PLA-chitosan-IM7 nano-particles on mice with ovarian cancer. Fluorescence animal models in vivo were successfully established (Fig. 5A), and the results indicated that PLA-chitosan-IM7 nano-particles could target the tumor cells and suppress their proliferation (Fig. 5B). A total of 35 days subsequent to PLA-chitosan-IM7 treatment, all animals were sacrificed, and the tumor tissues were removed and weighted. PLA-chitosan-IM7 could inhibit tumor growth effectively (Fig. 5C). Regarding the animals' behavior, the hair and mental state of the mice in the PLA-chitosan-IM7 group were

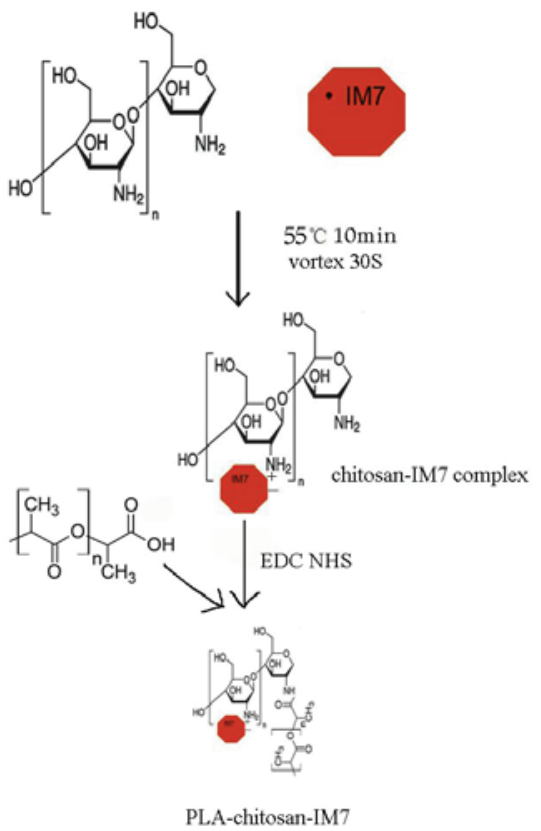

Figure 1. Process of preparation of PLA-chitosan-IM7 nano-particles. First, the anti-cluster of differentiation 44 antibody IM7 (200 $\mu 1)$ was added to a thiamine pyrophosphate solution (1 mg/ml, $\mathrm{pH} 7-9)$ and mixed well. Secondly, the mixture was added to a chitosan solution ( $\mathrm{pH} 4-6$ ), and by molecular reaction, chitosan-IM7 was formed. Thirdly, 1-ethyl-3-(3-dimethylaminopropyl) carbodiimide hydrochloride/N-hydroxysuccinimide was added to a PLA solution to activate the carboxyl moiety, and then the mixture was added to the chitosan-IM7 nano-particles. The reaction components were mixed thoroughly and were allowed to react for $15 \mathrm{~min}$ at room temperature. PLA, polylactic acid; mRNA, messenger RNA.

relatively better than those of mice in the other groups. As for the subcutaneous tumor model, similar results were obtained (Fig. 5D).

\section{Discussion}

HA and its receptor CD44 appear to be valid targets in numerous studies that had been designed to treat human ovarian cancers. However, their toxicity is their major issue, which limits the widespread use of these approaches (8). Therefore, it is important and meaningful to identify a new approach for cancer treatment. Nano-particles are easily absorbed by tissues and cells, and have been widely studied as a drug carrier in recent years. In addition, nano-particles have provided a good platform for cancer gene therapy based on their unique properties, including diverse surface chemistry, appropriate size scale and organ-specific pharmacokinetics (19). Chitosan has been long studied as a special drug delivery system, and one of the most important characteristics is to be developed to ionic gelatinization with polyanion $(20,21)$. Cai et al developed a gold-PEI nano-carrier with good transfection efficiency to deliver anti-Epstein-Barr virus (RBV) microRNA (miR)-BART7-3p and elicit its potential therapeutic effect. In vitro and in vivo data revealed that cell proliferation and tumor growth were effectively suppressed by anti-EBV-miR-BART7-3p transported by nano-particles, and the expression of relevant genes was modulated accordingly, indicating the feasibility of utilizing nano-particles to deliver anti-miR and silence endogenous EBV-miR-BART7-3p (22). 
A

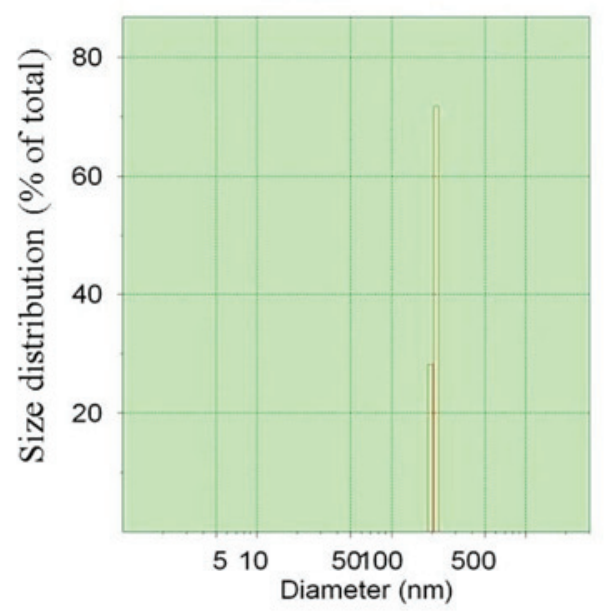

B

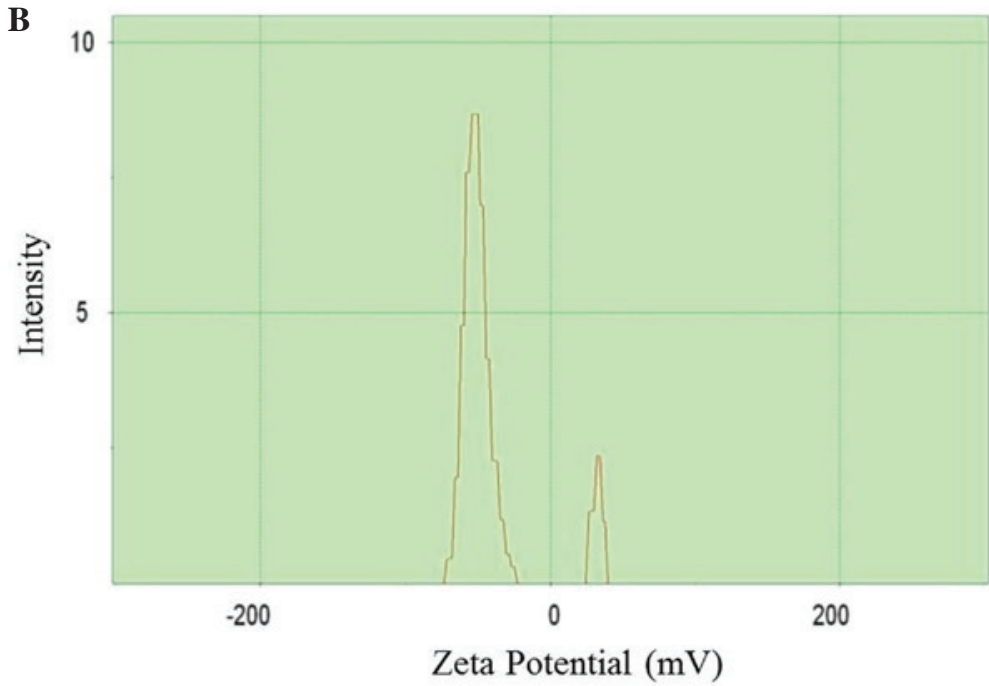

Figure 2. Transmission electron microscopy was used to observe (A) the diameter (nm) and (B) zeta potential (mV) of nano-particles. The diameter of the nano-particles was determined to be $100-500 \mathrm{~nm}$ (average value, $350 \mathrm{~nm}$ ) and the zeta potential was determined to vary between $-75 \mathrm{and}+45 \mathrm{mV}$.

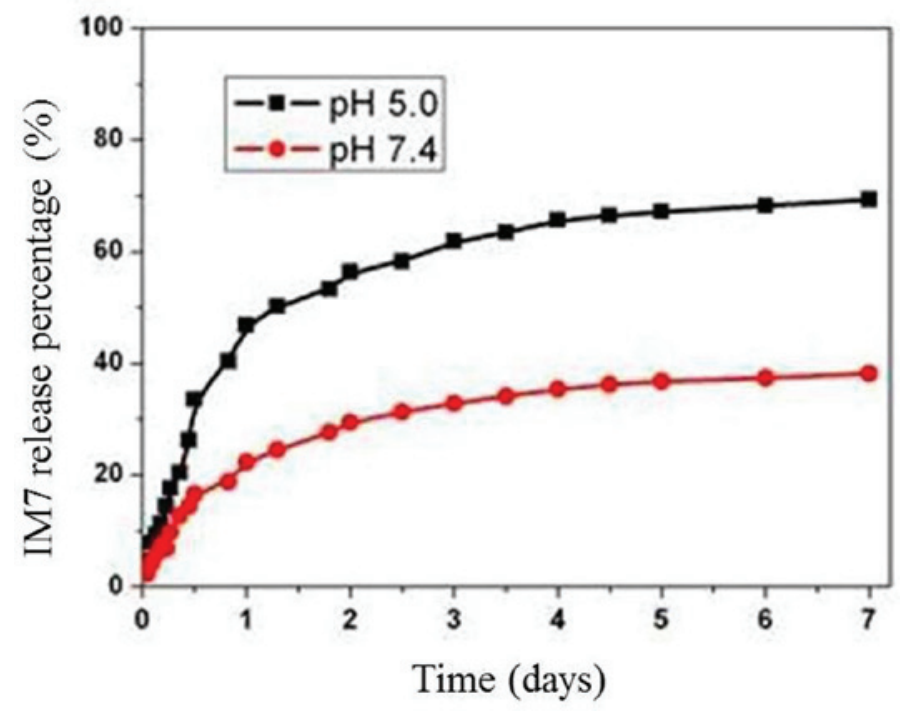

Figure 3. Release rate of PLA-chitosan-IM7 at neutral $(\mathrm{pH} 7.4)$ and acidic $(\mathrm{pH}$ 5.0) environments. The PLA-chiosan-IM7 release is slightly faster in an acid environment than in a neutral environment. A total of 3 days subsequent to PLA-chitosan-IM7 treatment, PLA-chitosan-IM7 was stable regardless the type of environment. PLA, polylactic acid.

In the present study, chitosan coated with PLA was used as the delivery system for IM7 in order to avoid its toxicity. Firstly, IM7 was added into a TPP solution (pH 7-9), and the mixture was then added into a chitosan solution ( $\mathrm{pH} 4-6)$. The nano-particles that carried the anti-CD44 antibody were prepared by reaction between TPP and chitosan. Upon preparation, TEM was used to detect the diameter and zeta potential of the particles, and it was observed that the former was $100-500 \mathrm{~nm}$ (mean value, $350 \mathrm{~nm}$ ) (Fig. 2A) and the latter was between -75 and $+45 \mathrm{mV}$, which requires further research. In addition, the stability of the anti-CD44 antibody IM7 carried with nano-particles was evaluated in acidic and neutral environments. The release rate was noticed to be slow and steady (Fig. 3). Secondly, the anti-tumor effect of PLA-chitosan-IM7 was assessed in vitro and in vivo. The human ovarian cell line HO-8910PM was used as the target cell, and it was observed that PLA-chitosan-IM7 could suppress the proliferation of cancer cells (Fig. 4). Then, an in vivo imaging system was used to investigate the anti-tumor effect of PLA-chitosan-IM7, and it was observed that PLA-chitosan-IM7 have relative organ-specific and improved anti-tumor growth effect (Fig. 5B and C). Additionally, the hair and mental state of the mice in the group treated with PLA-chitosan-IM7 were relatively better than those of the mice in the other groups.

Large efforts have been undertaken to improve the therapeutic efficacy of bioactive molecules, including cancer therapeutics. Nano-preparations and targeting the drug-loaded nano-carriers to the disease site to generate so-called 'magic bullets' are examples of the remarkable efforts that have been undertaken to design and develop strategies to improve the cellular uptake of therapeutic molecules and their delivery to an organelle of interest (23). The present study demonstrated 
The proliferation of HO-8910PM

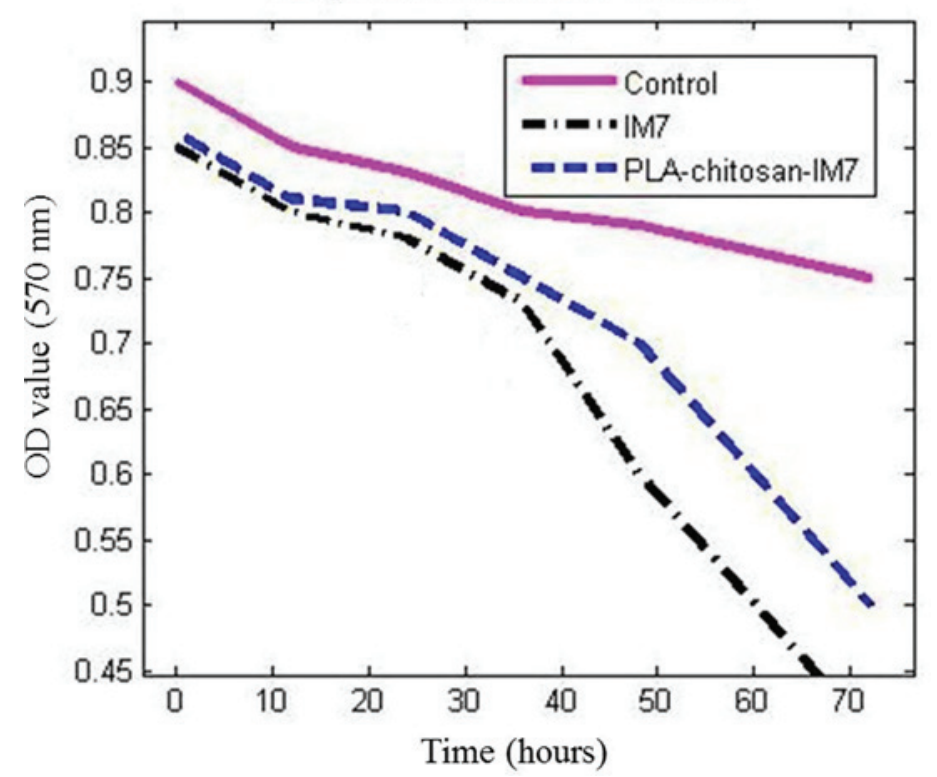

Figure 4. MTT assay was employed to analyze the proliferation of HO-8910PM cells. The PLA group was treated with PLA-chitosan-IM7 at 100 ng/ml; the IM7 group was treated with IM7 antibody at $100 \mathrm{ng} / \mathrm{ml}$; and the control group was treated with the same volume of saline. IM7 and PLA-chitosan-IM7 could both suppress the proliferation of tumor cells. In addition, the survival rate of the IM7 group was lower than that of the PLA-chitosan-IM7 group, suggesting that application of IM7 alone has higher toxicity. PLA, polylactic acid.

A

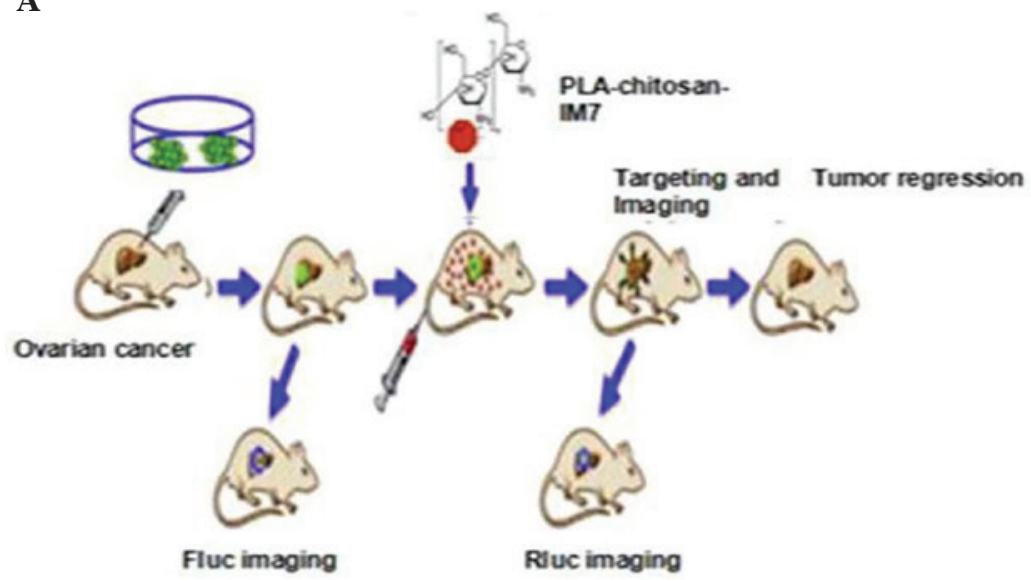

B

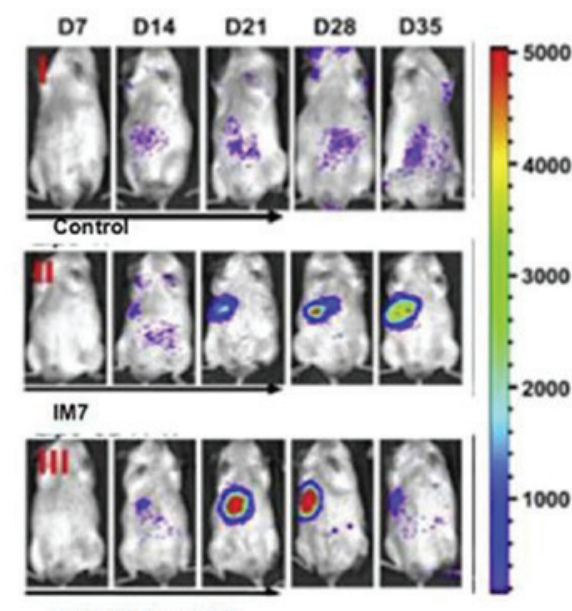

PLA-chitosan-IM7

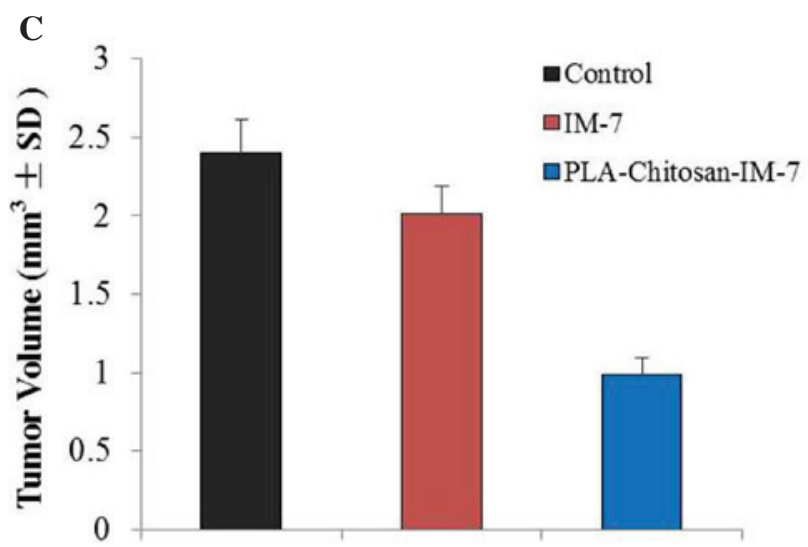

D 1200

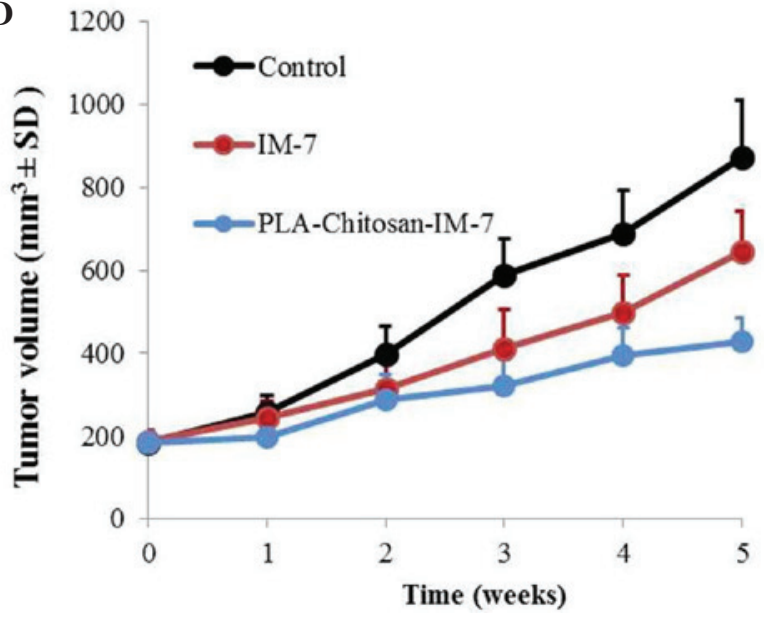

Figure 5. Effect of PLA-chitosan-IM7 nano-particles on mice with ovarian cancer. (A) Process of establishment of fluorescent animal models in vivo. (B) Animals subjected to live imaging. (C) Weight of tumors from animals subjected to different treatments. (D) Tumor volume was determined using calipers on the indicated times. Data are presented as the mean $\pm \mathrm{SD}, \mathrm{n}=10$. PLA, polylactic acid; SD, standard deviation. 
that PLA-chitosan-IM7 displays a good prospect in clinical application. However, future studies are required on its specificity, degradation and immune tolerance. The current study provided a new aspect of enhancing the effectiveness of cancer treatment while reducing its toxicity, which is of great use in tumor therapy, particularly in tumor biotherapy.

\section{References}

1. Siegel R, Naishadham D and Jemal A: Cancer statistics, 2013.CA Cancer J Clin 63: 11-30, 2013.

2. Bourguignon LY, Zhu H, Zhou B, Diedrich F, Singleton PA and Hung MC: Hyaluronan promotes Cd44V3-Vav2 interaction with Grb2-p185 (HER2) and induces Racl and Ras signaling during ovarian tumor cell migration and growth. J Biol Chem 276 48679-48692, 2001.

3. Carpenter PM and Dao AV: The role of hyaluronan in mesothelium-induced motility of ovarian carcinoma cells. Anticancer Res 23: 3985-3990, 2003.

4. Cannistra SA, Abu-Jawdeh G, Niloff J, Strobel T, Swanson L, Andersen $\mathrm{J}$ and Ottensmeier C: CD44 variant expression is a common feature of epithelial ovarian cancer: Lack of association with standard prognostic factors. J Clin Oncol 13: 1912-1921, 1995.

5. Ourguignon LY, Zhu H, Zhou B, Diedrich F, Singleton PA and Hung MC: Hyaluronan promotes Cd44V3-Vav2 interaction with Grb2-p185(HER2) and induces Racl and Ras signaling during ovarian tumor cell migration and growth. J Biol Chem 276 : 48679-48692, 2001

6. Bourguignon LY, Zhu H, Chu A, Iida N, Zhang L and Huang MC: Interaction between the adhesion receptor, CD44, and the oncogene product, p185HER2, promotes human ovarian tumor cell activation. J Biol Chem 272: 27913-27918, 1997.

7. Ourguignon LY, Zhu H, Shao L and Chen YW: CD44 interaction with c-Src kinase promotes cortactin-mediated cytoskeleton function and hyaluronic acid-dependdent ovarian tumor cell migration. J Biol Chem 276: 7327-7336, 2001.

8. Bourguignon LY, Peyrollier K, Gilad E and Brightman A: Hyaluronan-CD44 interaction with neural Wiskott-Aldrich syndrome protein (N-WASP) promotes actin polymerization and ErbB2 activation leading to beta-catenin nuclear translocation, transcriptional up-regulation, and cell migration in ovarian tumor cells. J Biol Chem 282: 1265-1280, 2007.

9. Bourugnon LY, Gilad E and Peyrollier K: Heregulin-mediated ErbB2-ERK signaling activates hyaluronan synthases leading to CD44-dependent ovarian tumor cell growth and migration. J Biol Chem 282: 19426-19441, 2007.

10. Zhu D and Bourguignon LY: Interaction between CD44 and the repeat domain of ankyrin promotes hyaluronic acid-mediated ovarian tumor cell migration. J Cell physiol 183: 182-195, 2000.
11. Tzuman YC, Sapoznik S, Granot D, Nevo N and Neeman M: Peritoneal adhesion and angiogenesis in ovarian carcinoma are inversely regulated by hyaluronan: The role of gonadoptropins. Neoplasia 12: 51-60, 2010.

12. Ween MP, Hummitzsch K, Roggers RJ, Oehler MK and Ricciardelli C: Versican induces a pro-metastatic ovarian cancer cell behavior which can be inhibited by small hyaluronan oligosaccharides. Clin Exp Metastasis 28: 113-125, 2011.

13. Lee H, Lee K and Park TG: Hyaluronic acid-paclitaxel conjugate micelles: Syhthesis, characterization, and antitumor activity. Bioconjug Chem 19: 1319-1325, 2008.

14. Cohen MS, Cai S, Xie Y and Forrest ML: A novel intralymphatic nanocarrier delivery system for cisplatin therapy in breast cancer with improved tumor efficacy and lower systemic toxicity in vivo. Am J Surg 198: 781-786, 2009.

15. Banzato A, Bibisse S, Rondina M, Renier D, Bettella F, Esposito G, Quintieri L, Meléndez-Alafort L, Mazzi U, Zanovello $\mathrm{P}$ and Rosato A: A paclitaxel-hyaluronan bioconjugate targeting ovarian cancer affords a potent in vivo therapeutic activity. Clin Cancer Res 14: 3598-3606, 2008.

16. Thompson CB, Shepard HM, O'Connor PM, Kadhim S, Jiang P, Osgood RJ, Bookbinder LH, Li X, Sugarman BJ, Connor RJ, et al: Enzymatic depletion of tumor hyaluronan induces antitumor responses in preclinical animal models. Mol Cancer Ther 9: 3052-3064, 2010.

17. Guedan S, Rojas JJ, Gros A, Mercade E, Cascallo M and Alemany R: Hyaluronidase expression by an oncolytic adenovrirus enhances its intratumoral spread and suppresses tumor growth. Mol Ther 18: 1275-1283, 2010.

18. Lee SJ, Min HS, Ku SH, Son S, Kwon IC, Kim SH and Kim K: Tumor-targeting glycol chitosan nanoparticles as a platform delivery carrier in cancer diagnosis and therapy. Nanomedicine Lond) 9: 1697-1713, 2014

19. Chen Y, Zhu X, Zhang X, Liu B and Huang L: Nanoparticles modified with tumor-targeting scFv deliver siRNA and miRNA for cancer therapy. Mol Ther 18: 1650-1656, 2010.

20. Sun X, Zeng L, Chen C, Huang Y, Han F, Xiao W, Liu S and Lu T: Comparing treatment outcomes of different chemotherapy sequences during intensity modulated radiotherapy for advanced $\mathrm{N}$-stage nasopharyngeal carcinoma patients. Radiat Oncol 8: $265,2013$.

21. Elbakry A, Zaky A, Liebl R, Rachel R, Goepferich A and Breunig M: Layer-by-layer assembled gold nanoparticles for siRNA delivery. Nano Lett 9: 2059-2064, 2009.

22. Cai L, Li J, Zhang X, Lu Y, Wang J, Lyu X, Chen Y, Liu J, Cai H, Wang Y and Li X: Gold nano-particles (AuNPs) carrying anti-EBV-miR-BART7-3p inhibit growth of EBV-positive nasopharyngeal carcinoma. Oncotarget 6: 7838-7850, 2015.

23. Biswas $S$ and Torchilin VP: Nanopreparations for organelle-specific delivery in cancer. Adv Drug Deliv Rev 66: 26-41, 2014. 